\title{
CFTR deficiency causes cardiac dysplasia during zebrafish embryogenesis and is associated with dilated cardiomyopathy
}

\section{Yanyan Liu}

Sichuan University

Ziyuan Lin

Sichuan University

Mingfeng Liu

Sichuan University

Huijuan Liao

Sichuan University

\section{Yan Chen}

Sichuan University

Xiaohu Zhang

Sichuan University

Hsiao Chang Chan

Sichuan University

Bin Zhou

Sichuan University

Li Rao

Sichuan University

Huaqin Sun ( $\nabla$ sunhuaqin@scu.edu.cn )

Sichuan University https://orcid.org/0000-0002-4548-4657

\section{Research article}

Keywords: CFTR, Cardiac development, Dilated cardiomyopathy, Channel defect

Posted Date: March 23rd, 2020

DOI: https://doi.org/10.21203/rs.3.rs-18648/v1

License: (c) (1) This work is licensed under a Creative Commons Attribution 4.0 International License.

Read Full License 
Version of Record: A version of this preprint was published at Mechanisms of Development on September 1st, 2020. See the published version at https://doi.org/10.1016/j.mod.2020.103627. 


\section{Abstract}

Background: Mutations in the CFTR gene cause cystic fibrosis (CF) with myocardial dysfunction. However, it remains unknown whether CF-related heart disease is a secondary effect of pulmonary disease, or an intrinsic primary defect in the CF heart.

Results: Here, we used a zebrafish model, which lacks lung tissue, to investigate the role of CFTR in cardiogenesis during embryonic development. Our findings demonstrate that a loss of CFTR impairs cardiac development from the cardiac progenitor stage of heart development, resulting in cardiac looping defects, a dilated atrium, pericardial edema, and a decrease in heart rate. Furthermore, we found that cardiac development is perturbed in wild type embryos treated with a gating specific Cftr channel inhibitor, CFTRinh-172, at the blastula stage of development, but not with treatment at later stages. Gene expression analysis of blastulas indicated that transcript levels, including mRNAs associated with cardiovascular diseases, were significantly altered in embryos derived from cftr mutants relative to controls. To evaluate the role of CFTR in human heart failure, we performed a genetic association study on individuals with dilated cardiomyopathy and found that CFTR containing I556V mutation, which causes a channel defect, is associated with the disease. Similar to well-studied channel-defective CFTR mutants, CFTR I556V mRNA failed to restore cardiac dysplasia in mutant embryos.

Conclusions: The present study reveals an important role for the CFTR ion channel in regulating cardiac development during early embryogenesis, supporting the hypothesis that CF-related heart disease results from an intrinsic primary defect in the CF heart.

\section{Background}

Mutations in cystic fibrosis transmembrane conductance regulator (CFTR) have been shown to cause cystic fibrosis (CF) [1, 2], and the CF hallmark is electrolyte and fluid transport defect resulting in a multitude of clinical manifestations [1, 2]. Defective ion channel function of CFTR due to the most common mutation $\triangle F 508$ in the CFTR gene has been shown to underlie the pathogenesis of disease conditions, such as CF related abnormal gonad function and infertility [3-5], CF-related diabetes [6], obstructive lung disease $[7,8]$ and pancreas exocrine deficiency [9].

The feature of $\mathrm{CF}$ is developmental organ failure induced by the progress of scarring and fibrosis. The Caucasian population is affected mainly as a fatal chronic autosomal recessive systemic disorder[1012]. Importantly, pathological, experimental, and clinical evidence reported by a number of clinical studies and case reports in the past four decades indicates heart disease with myocardial dysfunction in CF, which affects systolic and diastolic heart function and possibly starts from childhood[11]. Early subclinical changes detected by transthoracic echocardiogram (TTE) and sensitive echocardiographic modalities [13-15] have provided evidence of abnormal myocardial function in CF, leading to the possibility of there being a CF-related cardiomyopathy $[11,15]$. 
Because zebrafish genome sequence program has been completed and the high conservation of gene function between zebrafish and humans is uncovered, zebrafish have become an attractive model for studying the function of human disease-related gene variants. Especially, zebrafish model can provide novel mechanism insights into human cardiovascular disease [16].

The current dogma considers that heart dysfunction in $\mathrm{CF}$ is a secondary defect that results from the initial pulmonary disease; however, the presence of CFTR in cardiac myocardia and its involvement in the regulation of cardiomyocyte contraction has prompted a debate as to whether CF-associated heart disease is caused by an intrinsic primary defect of the heart [17].

Here, we demonstrate that Cftr mutation leads to aberrant cardiac development, resulting in myocardial dysplasia in zebrafish embryos. Furthermore, we demonstrate that defects in CFTR are associated with dilated cardiomyopathy, suggesting that CFTR plays an essential role in myocardial development and function. Thus, our results provide support for the argument that CF-associated heart disease is likely to be caused by an intrinsic, primary cardiac defect.

\section{Results}

$2.1 \mathrm{cftr}$ mutation leads to defects in cardiogenesis beginning at the stage where cardiac progenitor cells emerge

To determine whether CFTR plays an important role in cardiac development during zebrafish embryogenesis, we first performed morphological phenotypic analysis on two existing cftr mutant zebrafish lines, cftr ${ }^{\text {scu101 [18] (http://zfin.org/action/feature/view/ZDB-ALT-181127-1) and cftr }}{ }^{\text {scu102 [19] }}$ (http://zfin.org/action/feature/view/ZDB-ALT-190307-1). cftr" ${ }^{\text {scu101 }}$ carried a 9-bp deletion, which was predicted to delete the start codon of $\mathrm{Cftr}$; $\mathrm{cftr}^{\mathrm{scu} 102}$ carried a 2-bp deletion in Exon 6, causing frameshifts leading to premature stop codons at 219 AA. This revealed that embryos from the two cftr mutant lines similarly develop pericardial edema by $60 \mathrm{hpf}$ (Fig. 1A), accompanied by a lower heart rate (Fig. 1B) and a dramatic reduction in systolic function (Fig. 1C) as measured by ventricular fractional shortening

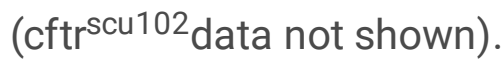

The $\mathrm{cftr}^{\text {scu101 }}$ mutant line was used for the following analyses. To further determine the effect of cftr mutation on heart development, we investigated the expression levels of several common cardiac markers by ISH in cftr ${ }^{\text {scu101 }}$ embryos. At the 8-somite stage (13 hpf), when cardiogenic differentiation is initiated, the expression of the cardiac mesoderm marker nkx2.5 was dramatically affected (Fig. 1D). Staining for the two populations of $\mathrm{nkx} 2.5^{+}$cardiac precursors, which lie adjacent to the midline, was not only reduced in intensity (Fig. 1E), but their distance from one another was also increased (Fig. 1F). These data suggest that the specification and migration of cardiac mesoderm is greatly impaired in cftr mutants. 
To further investigate the effect of cftr mutation on cardiac morphogenesis in zebrafish, cardiomyocyte marker myl7 (formerly indicated as cmlc2) expression was examined by ISH. In WT embryos, the heart tube had formed at $24 \mathrm{hpf}$, that is, cardiac progenitors were specified correctly and extended from the midline to the region under the left eye to form the linear heart tube. However, consistent with a previously described role for $\mathrm{cftr}$ in the establishment of embryonic laterality [20], $\mathrm{cftr}^{\mathrm{scu} 101}$ had heart tubes located at the midline and to the right, resulting in the formation of a defective heart tube by $48 \mathrm{hpf}$ (Fig. 1G). Because of the variation existence of defective heart phenotype among larvae, the angle between the longitudinal axes of the ventricle and atrium was measured to demonstrate the looping defect. Results indicated that the statistical looping angle (average $3^{\circ}$ ) of $\mathrm{cftr}^{\text {scu101 }}$ was decreased significantly when compared to WT embryos (average $21^{\circ}$ ) (Fig. $1 \mathrm{H}$ ). Furthermore, cftr ${ }^{\text {scu101 }}$ embryos also showed markedly dilated atria (Fig. 11).

We also assessed the expression of the atrioventricular boundary marker genes bmp4 (Fig. $1 \mathrm{~J}$ and $\mathrm{K}$ ) and has2 (Fig. $1 \mathrm{~L}$ and $\mathrm{M}$ ) in the cftr mutants, and found that the expression of these two marker genes was also dramatically weakened in $\mathrm{cftr}^{\mathrm{scu} 101}$ embryos. Taken together, these results suggest that cftr plays an essential role in early heart morphogenesis, particularly at the stage of cardiogenic differentiation.

2.2 The blastula stage is the key temporal window of cftr function on cardiogenesis

To further characterize the role of cftr ion channel function during cardiogenesis, we investigated the effect of the CFTR inhibitor CFTRinh-172 on WT embryos at different time points. CFTRinh-172 is a potent and specific inhibitor of the CFTR channel that was identified by high-throughput screening [21, 22]. During the blastula stage ( $5 \mathrm{hpf})$, cardiac progenitor cells are located bilaterally in the lateral marginal zone [16]. We first treated WT embryos at 3-5 hpf with $10 \mu \mathrm{m}$ CFTRinh-172, a dose widely used to inhibit cftr ion channel activity [23]. Interestingly, the embryos treated by CFTRinh-172 at this time point showed cardiac dysplasia similar to $\mathrm{cftr}^{\mathrm{scu} 101}$ (Fig. 2A-H).

Next, we treated WT embryos at the gastrula stage, when cardiac progenitor cells move dorsally towards the mid-line before migrating to the anterior lateral plate mesoderm (ALPM) [16]. However, expression and localization of the cardiac marker nkx2.5 did not show significant abnormality when embryos were treated at either the early (treatment at 6-8 hpf) and late (treatment at 8-10 hpf) gastrula stages (Supplemental Fig. 1A-F). In brief, these results indicate that the blastula stage is the stage at which cftr affects cardiogenesis.

2.3 RNA-seq analysis reveals significant changes in the expression levels of factors essential for cardiogenesis in $\mathrm{cftr}^{\mathrm{scu} 101}$ embryos at the blastula stage

To uncover the molecular mechanisms of cftr regulation during cardiogenesis, and to quantify the dynamic changes across the entire transcriptome, we performed RNA-seq analysis in WT and cftr mutant zebrafish embryos at the blastula stage ( $5 \mathrm{hpf}$ ). Results showed that cftr mutants differentially expressed 1334 transcripts (412 up-regulated and 922 down-regulated) (Supplemental Table 1). To characterize the 
function of these altered mRNAs, KEGG pathway-based classification analysis on the ontology of cellular processes, environmental information processing, genetic information processing, human diseases, metabolism, and organismal systems was performed, and revealed a widely different distribution in cftr $^{\text {scu101 }}$ mutant embryos compared to WT (Fig. 3A and Supplemental Table 2).

Of note, human disease-based enrichment results found that transcripts for genes involved in cardiovascular diseases, including dilated cardiomyopathy (DCM) and hypertrophic cardiomyopathy (HCM) (Fig. 3B and C, Supplemental Tables 3 and 4), were significantly affected in cftrscu101 mutants. Then, we selected 10 representative genes with significant expression change for further identification, quantitative PCR results showed that all detected genes demonstrated similar expression pattern in embryos (Fig. 3D). In conclusion, gene expression analysis comparing WT and cftr mutant embryos indicated that cftr deficiency induces significant changes in transcript levels, resulting in the altered expression of many mRNAs critical to cardiovascular diseases.

2.4 CFTR I556V mutation in human dilated cardiomyopathy

Due to the severe cardiomyopathy and alteration of cardiovascular disease-related mRNAs resulting from the loss of cftr in zebrafish, as well as to provide more convincing evidence for the role of CFTR in myocardial development, we considered that whether CFTR was associated with human heart trouble. So, the potential involvement of CFTR in a Chinese population of dilated cardiomyopathy was evaluated.

Fortunately, we enrolled 1 propositus (see Supplemental Table 5 for clinical information and Supplemental Fig. 2 for sequencing data) with family history of diagnosed idiopathic dilated cardiomyopathy for CFTR mutation screening by whole-exome sequencing. Interestingly, we identified a heterozygous missense mutation ( $A \rightarrow G$ at position 1666, resulting in the amino acid change $1556 \mathrm{~V}$ ) in this propositus. Furthermore, this CFTR I556V mutation was not identified in over gender/age/geography/ethnicity matched 600 healthy controls, demonstrating a highly significant association between the CFTR mutation and dilated cardiomyopathy through exact Fisher's test analysis $(P<0.001)$.

2.5 CFTR I556V mutation fails to rescue the cardiac dysplasia in zebrafish cftr mutant model

The CFTR I556V mutation was initially reported in a French male who presented with asthma-like bronchopathy and chronic diarrhea [24, 25]. 1556V and a second mutation, F508del, are the most common types of CFTR mutations in Chinese patients with CF and congenital bilateral absence of the vas deferens (CBAVD) $[26,27]$. Importantly, compared with WT, open probability (Po) of $\mathrm{Cl}^{-}$channel function was significantly reduced in the presence of I556V (by 34\%) [28]. However, the association of the CFTR I556V mutation with cardiac development and dilated cardiomyopathy has not yet been investigated.

To investigate the function of CFTR $1556 \mathrm{~V}$ mutation in the heart, we sought to restore the cardiac dysplasia in cftr mutants by by injecting human CFTR $1556 \mathrm{~V}$ mRNAs into mutant embryos. CFTR wildtype or channel-defective human CFTR ( $\triangle$ F508 and G551D) mRNAs were injected in parallel for 
comparison. The CFTR $\triangle \mathrm{F} 508$ mutation results in protein misfolding and subsequent protein retention in the endoplasmic reticulum (ER), with a negligible amount of mutant CFTR reaching the plasma membrane, leading to impaired ion channel function [29], while CFTR G551D is a well-known mutation causing gating defect in CFTR channel function [30,31].

As with the channel-defective $\Delta$ F508 and G551D mutants, CFTR I556V failed to restore the cardiac dysplasia in cftr mutants, as evidenced by altered nkx2.5 expression at $13 \mathrm{hpf}$ (Fig. 4A-C), myl7 expression at $48 \mathrm{hpf}$ (Fig. 5A-C), heart rate (Fig. 6A) and systolic function at $60 \mathrm{hpf}$ (Fig. 6B). In contrast, WT CFTR mRNA markedly restored the cardiogenesis in offspring embryos derived from the mutant line. Interestingly, a $\triangle \mathrm{PDZ}$ mutant, which lacks the protein interaction module but has intact channel function, also rescued the cardiogenic defects. Taken together, these results, along with those demonstrating impaired cardiogenesis in the presence of CFTRinh-172, indicate that cftr ion channel activity is essential to cardiac development.

\section{Discussion}

Many reports have shown the association of the myocardium structural changes in CF[11]. Here, we used a zebrafish model without lung tissue to demonstrate that cftr deficiency impairs cardiac development at early embryonic stages, resulting in cardiac looping defects, a dilated atrium, pericardial edema, and a decrease in heart rate. Especially, we find a small number but significant altered expression of genes associated with cardiac dysfunction are enriched in cftr mutant embryos revealed by RNA-seq analysis, giving the link between embryonic cardiac deficiency caused by cftr mutant and cardiac defects in individuals with CF and supporting the argument that the CF-related heart disease is an intrinsic primary defect in the CF heart.

Dilated cardiomyopathy (DCM) is a primary cardiomyopathy characterized by unilateral or bilateral cardiac expansion, myocardial contractile dysfunction, and thinning of the ventricular wall. Previous studies have suggested that DCM is caused by multiple factors such as genetics, autoimmune abnormalities, viral infections, poisons, or drugs. However, with the development of molecular genetics and whole-genome linkage analysis techniques, recent studies have found that more than $40 \%$ of DCM patients have a familial genetic predisposition [32]. Familial dilated cardiomyopathy can be diagnosed in a patient if two or more immediate family members have DCM, or if sudden cardiac death occurs in a young person. The most important genetic model of DCM is autosomal dominant inheritance.

The electrical activity of cardiomyocytes depends on the normal function of various ion channels. Sodium is an important ion current in cardiomyocytes, as it mediates the rapid ascending phase of the action potential of cardiomyocytes, and determines the excitatory conduction of these cells. CFTR regulates chloride transport while also regulating sodium transport. The activity of the epithelial sodium channel $(\mathrm{ENaC})$ is dependent on CFTR and increases with increasing CFTR activity. Electrophysiological experiments have been used to demonstrate synergy between the two ion channels, and increased chloride transport by CFTR activation also enhances the ability of sodium ions to pass through the ENaC. 
Furthermore, studies have found that ENaC activation requires the involvement of intact CFTR, and its expression is dependent on the help of CFTR. Thus, CFTR deficiency also leads to epithelial sodium ion abnormalities [33].

CFTR is well known as a phosphorylation-dependent chloride channel, and in addition to the apical membranes widely present in various epithelial cells, such as the respiratory tract and the intestine, CFTR expression has also been observed in the myocardium. Recordings of cardiomyocytes taken at the whole cell level have shown that chloride current activation by cyclic adenosine monophosphate-PKA $\left(\mathrm{I}_{\mathrm{Cl} \text {.PKA }}\right)$, PKC ( $\left.I_{\mathrm{CI} . \mathrm{PKC}}\right)$, and by purinergic receptors (extracellular triphosphate adenine ATP; $\mathrm{I}_{\mathrm{Cl} . \mathrm{ATP}}$ ) is mediated by CFTR $[34,35]$. In CFTR knockout (KO) mouse model, cardiomyocytes with disrupted CFTR function require CaMKII and $\mathrm{Ca}^{2+}$-activated $\mathrm{Cl}^{-}$channel activity to maintain contraction rate[36]. In a mouse model of CF $(\triangle \mathrm{F} 508), \mathrm{CFTR}$ mutation leads to left ventricular (LV) remodeling with alteration of cardiac and aortic functions in the absence of lung disease[37]. In this study, we indeed found that the CFTR I556V mutation was associated with impaired channel function in a propositus with family history of dilated cardiomyopathy, providing further evidence for the between CFTR and heart disease.

\section{Conclusions}

We used a zebrafish model, which lacks lung tissue, to demonstrate that a loss of CFTR impairs cardiac development from the cardiac progenitor stage of heart development, resulting in cardiac looping defects, a dilated atrium, pericardial edema, and a decrease in heart rate. Gene expression analysis of blastulas indicated that transcript levels, including mRNAs associated with cardiovascular diseases, were significantly altered in embryos derived from cftr mutants relative to controls. Furthermore, we found that CFTR containing $1556 \mathrm{~V}$ mutation, which causes a channel defect, is associated with dilated cardiomyopathy by a genetic association study, and CFTR I556V mRNA failed to restore cardiac dysplasia in mutant embryos. The present study reveals an important role for the CFTR ion channel in regulating cardiac development during early embryogenesis, supporting the hypothesis that CF-related heart disease results from an intrinsic primary defect in the CF heart.

\section{Methods}

\subsection{Zebrafish care}

All experiments in this study were conducted in accordance with the "Guide for the Care and Use of Laboratory Animals" (Eighth Edition, 2011. ILARCLS, National Research Council, Washington, D.C.) and were approved by the Animal Care and Use Committee of West China Second University Hospital, Sichuan University (Approval ID: HXDEYY20131021). The standard care of zebrafish followed the guidelines from China Zebrafish Resource Center (http://www.zfish.cn/) and THE ZEBRAFISH BOOK (https://zfin.org/zf_info/zfbook/zfbk.html). In the laboratory, as zebrafish older than 18 months begin to age, their spawning is reduced, their immunity is reduced, they are susceptible to bacteria, or they can grow tumors, which can be euthanized in time dealing with humane endpoints and pain management. 
The euthanasia method of zebrafish was rapid cooling performed as previously described[38, 39] and approved by IACUC (Institutional Animal Care and Use Committee). Briefly, place the fish in a small volume of water in a container of adequate size, and fish must not come into direct contact with ice; add four times the volume of ice on top of water; monitor water temperature with a thermometer; keep fish in chilled water until 20 minutes after orientation loss and operculum movement cessation; remove fish and store in freezer until appropriate carcass disposal. Our research study also covers the 3Rs (refinement, replacement, and reduction).

5.2 Zebrafish embryo manipulation, in situ hybridization, In vitro synthesis of RNA, microinjection, fractional shortening and cardiac looping angle measurement

Wild type (WT) zebrafish embryos from the AB strain were used, while cftr mutant zebrafish lines $\mathrm{cftr}^{\text {scu101 }}$ and $\mathrm{cftr}^{\mathrm{scu} 102}$ were previously established and maintained in the lab[18, 19]. Embryos manipulation, whole-mount in situ hybridization (ISH), in vitro synthesis of RNA, microinjection (injection dose was $\sim 50$ pg mRNA per embryo) and statistics were carried out as previously described[18, 19]. Fractional shortening of the ventricles and heart rate was calculated using software from the Noldus company (https://www.noldus.com/). Measurement for cardiac looping angle at $48 \mathrm{hpf}$ was done as Xiyang Peng et al (2016)[40] and Priya Choudhry et al (2013)[41] described.

5.3 Analysis of the transcriptome

We screen and breed homozygous cftr mutant adult fish in our lab, and the $\mathrm{cftr}^{\mathrm{scu} 101}$ embryos were generated from the homozygous cftr mutant adult parents. We collected part of $\mathrm{cftr}^{\text {scu101 }} \mathrm{embryos}^{\mathrm{for}}$ genotyping, and the rest of 100 embryos were sent to RNA extraction and RNA seq experiments. Embryos were collected from WT and cftr mutant lines and sent to Chengdu Life Baseline Technology Co., LTD for transcriptomic analysis by RNA-seq as previously described[42].

\subsection{Reagents and constructs}

CFTRinh-172 (Catalog No. S7139) was from Selleck and diluted in DMSO. At the beginning of the blastula stage ( 3 hours post fertilization (hpf)) or beginning of the gastrula stage (6 hpf) or midgastrulation stage ( $8 \mathrm{hpf}$ ), embryos (30 embryos in a well of 6-well plate with $3 \mathrm{ml}$ culture water) were treated with CFTRinh-172 for 2 hours and then subjected to whole-mount ISH. All the treatment and ISH assay was performed with three independent biological replicates. WT CFTR plasmid was previously generated and maintained in the lab[19], while $\Delta$ F508, G551D, and I556V mutant plasmids were constructed using the KOD-Plus-Mutagenesis Kit (TOYOBO).

5.5 Gray scale measurement.

Whole-mount ISH images were taken under same magnification and exposure settings by Nikon SMZ1270 microscopy and standardized them with same software setting. Signal strength gray scale of whole-mount ISH images was measured by ImageJ software.

5.6 Quantitative real time RT-PCR (qPCR) analysis and statistics. 
Quantitative real time RT-PCR and related statistics were performed as previously described[18]. The primers used to detect cacna2d2a were "GTCCAGTACGGATCAGACTTC" and

"TGTTTGGAGTCCGGTGTGAC"; atp2a2a were "CACTGACACATAGTTCTTTTTGGG" and

"TCTGCAGGCAACTCGTTAGG"; hip1ra were "CGCGCTCGTGTTGATTATTGA" and

"TCGCAGGAGATTTGCCATGT"; CT990561.2 were "CAAGAACAAATGGCTGTCGG" and

"CGCAGTATGACGCTGTGGAA"; itgbl1 were "ATGCCAGGTCACAGAACCAG" and

"TTTGCTGGAGAGCGAACACT"; itga10 were "CAGTTGACTGAGGCAGACGA" and

"ACCGCTCTCACACTCAAGAAA"; si:ch211-211 k8.4 were "ATCATCACAGACCGACATCCTC" and

"CATTTACGCAACTTCTCACAACC"; tpm4b were "GAAGCGGATCCAGATTTGCG" and

"ATCTCCTTCAGCTTTCTCGC"; itgb1b.1 were "AGACCTGCATATAGGTTTTGGT" and

"TGTCTGTCAAGCTCAGGACG"; mamdc2b were "AGAACAGAGCCGTCGTTTGAT" and

"AGACCCTGCTCCTGTGGTAT"; F0904953.1 (smyhc2) were "AGGCCAATTCTAACCTGGGC" and

"CTTCGTCAGCCCCCTTCTTG"; $\beta$-actin (housekeeping gene) were "ATGAGTCTGGCCCATCCATC" and "CCTTTGCCAGTTTCCGCATC".

\section{Abbreviations}

CF: Cystic fibrosis; CFTR: Cystic fibrosis transmembrane conductance regulator; ABC: ATP-binding cassette; TTE: transthoracic echocardiogram; WT: Wild type; ISH: in situ hybridization; FRKM: per million mapped reads; SD: standard deviation; CBAVD: congenital bilateral absence of the vas deferens; Po: open probability; ER: endoplasmic reticulum; DCM: Dilated cardiomyopathy; ENaC: epithelial sodium channel; KO: knockout; LV: left ventricular

\section{Declarations}

\section{Ethics approval and consent to participate}

All experiments in this study were conducted in accordance with the "Guide for the Care and Use of Laboratory Animals" (Eighth Edition, 2011. ILARCLS, National Research Council, Washington, D.C.) and were approved by the Animal Care and Use Committee of West China Second University Hospital, Sichuan University (Approval ID: HXDEYY20131021).

Peripheral blood samples from dilated cardiomyopathy (DCM) patients and healthy controls were obtained from West China Hospital and West China Second University Hospital, and work involving the use of these samples was approved by these medical institutions (Approval ID: 2018, No. 32). Written informed consent was obtained from the patients and healthy control donors for publication of this study and any accompanying images. A copy of the written consent is available for review by the Editor of this journal. All procedures performed in studies involving human participants were in accordance with the ethical standards of the institutional and/or national research committee and with the 1964 Helsinki declaration and its later amendments or comparable ethical standards. 


\section{Consent for publication}

Not applicable.

\section{Availability of data and material}

All data generated and analyzed during this study are included in this published article and its additional files.

\section{Competing interests}

All the authors listed declare no competing interests and have approved the manuscript that is enclosed.

\section{Funding}

This research was supported by grants from National Natural Science Foundation of China (81670346 and 81200339), China Postdoctoral Science Foundation (20110491723), Young Teacher foundation of Sichuan University (2011SCU11040), National Basic Research Program of China (2012CB944903) and National 973 project (2013CB967404). The funders had no role in the design of the study and collection, analysis, and interpretation of data and in writing the manuscript.

\section{Authors' contributions}

H.S., L.R., B.Z., H.C.C. and X.Z. conceived and designed the experiments; H.S., Y.L., M.L. and Z.L. performed the experiments, with the assistance of H. L. and Y. C.; L.R. and B.Z. provided DNA samples and related supports; H.S., H.C.C. and X.Z. analyzed the data; H.S. and B.Z. wrote the paper. All authors have read and approved the manuscript.

\section{Acknowledgements}

Not applicable.

\section{References}

1. Riordan JR: CFTR function and prospects for therapy. Annu Rev Biochem 2008, 77:701-726.

2. Quinton PM: Physiological basis of cystic fibrosis: a historical perspective. Physiological reviews 1999, 79(1 Suppl):S3-S22.

3. Xu WM, Shi QX, Chen WY, Zhou CX, Ni Y, Rowlands DK, Yi Liu G, Zhu H, Ma ZG, Wang XF et al: Cystic fibrosis transmembrane conductance regulator is vital to sperm fertilizing capacity and male fertility. Proc Natl Acad Sci U S A 2007, 104(23):9816-9821.

4. Lu YC, Chen H, Fok KL, Tsang LL, Yu MK, Zhang XH, Chen J, Jiang X, Chung YW, Ma AC et al: CFTR mediates bicarbonate-dependent activation of miR-125b in preimplantation embryo development. Cell Res 2012, 22(10):1453-1466. 
5. Chen H, Guo JH, Lu YC, Ding GL, Yu MK, Tsang LL, Fok KL, Liu XM, Zhang XH, Chung YW et al: Impaired CFTR-dependent amplification of FSH-stimulated estrogen production in cystic fibrosis and PCOS. J Clin Endocrinol Metab 2012, 97(3):923-932.

6. Guo JH, Chen H, Ruan YC, Zhang XL, Zhang XH, Fok KL, Tsang LL, Yu MK, Huang WQ, Sun X et al: Glucose-induced electrical activities and insulin secretion in pancreatic islet beta-cells are modulated by CFTR. Nature communications 2014, 5:4420.

7. Johannesson B, Hirtz S, Schatterny J, Schultz C, Mall MA: CFTR regulates early pathogenesis of chronic obstructive lung disease in betaENaC-overexpressing mice. PLoS One 2012, 7(8):e44059.

8. Pezzulo AA, Tang XX, Hoegger MJ, Alaiwa MH, Ramachandran S, Moninger TO, Karp PH, WohlfordLenane $\mathrm{CL}$, Haagsman HP, van Eijk $\mathrm{M}$ et al: Reduced airway surface $\mathrm{pH}$ impairs bacterial killing in the porcine cystic fibrosis lung. Nature 2012, 487(7405):109-113.

9. Wilschanski M, Novak I: The cystic fibrosis of exocrine pancreas. Cold Spring Harbor perspectives in medicine 2013, 3(5):a009746.

10. Davies JC, Alton EW, Bush A: Cystic fibrosis. BMJ 2007, 335(7632):1255-1259.

11. Labombarda F, Saloux E, Brouard J, Bergot E, Milliez P: Heart involvement in cystic fibrosis: A specific cystic fibrosis-related myocardial changes? Respir Med 2016, 118:31-38.

12. Bright-Thomas RJ, Webb AK: The heart in cystic fibrosis. $J R$ Soc Med 2002, 95 Suppl 41:2-10.

13. Ozcelik N, Shell R, Holtzlander M, Cua C: Decreased right ventricular function in healthy pediatric cystic fibrosis patients versus non-cystic fibrosis patients. Pediatr Cardio/ 2013, 34(1):159-164.

14. Labombarda F, Pellissier A, Ellafi M, Creveuil C, Ribault V, Laurans M, Guillot M, Bergot E, Grollier G, Milliez P et al: Myocardial strain assessment in cystic fibrosis. J Am Soc Echocardiogr 2011, 24(9):1037-1045.

15. Sellers ZM, McGlocklin L, Brasch A: Strain rate echocardiography uncovers subclinical left ventricular dysfunction in cystic fibrosis. J Cyst Fibros 2015, 14(5):654-660.

16. Bakkers J: Zebrafish as a model to study cardiac development and human cardiac disease. Cardiovasc Res 2011, 91(2):279-288.

17. Sayyid ZN, Sellers ZM: Technological advances shed light on left ventricular cardiac disturbances in cystic fibrosis. J Cyst Fibros 2017, 16(4):454-464.

18. Liao H, Chen Y, Li Y, Xue S, Liu M, Lin Z, Liu Y, Chan HC, Zhang X, Sun H: CFTR is required for the migration of primordial germ cells during zebrafish early embryogenesis. Reproduction 2018 , 156(3):261-268.

19. Sun H, Wang Y, Zhang J, Chen Y, Liu Y, Lin Z, Liu M, Sheng K, Liao H, Tsang KS et al: CFTR mutation enhances Dishevelled degradation and results in impairment of Wnt-dependent hematopoiesis. Cell Death Dis 2018, 9(3):275.

20. Navis A, Marjoram L, Bagnat M: Cftr controls lumen expansion and function of Kupffer's vesicle in zebrafish. Development 2013, 140(8):1703-1712. 
21. Ma T, Thiagarajah JR, Yang H, Sonawane ND, Folli C, Galietta LJ, Verkman AS: Thiazolidinone CFTR inhibitor identified by high-throughput screening blocks cholera toxin-induced intestinal fluid secretion. J Clin Invest 2002, 110(11):1651-1658.

22. Taddei A, Folli C, Zegarra-Moran O, Fanen P, Verkman AS, Galietta LJ: Altered channel gating mechanism for CFTR inhibition by a high-affinity thiazolidinone blocker. FEBS Lett 2004, 558(1-3):5256.

23. Li H, Findlay IA, Sheppard DN: The relationship between cell proliferation, $\mathrm{Cl}$ - secretion, and renal cyst growth: a study using CFTR inhibitors. Kidney Int 2004, 66(5):1926-1938.

24. Ngiam NS, Chong SS, Shek LP, Goh DL, Ong KC, Chng SY, Yeo GH, Goh DY: Cystic fibrosis transmembrane conductance regulator (CFTR) gene mutations in Asians with chronic pulmonary disease: a pilot study. J Cyst Fibros 2006, 5(3):159-164.

25. Sakamoto H, Yajima T, Suzuki K, Ogawa Y: Cystic fibrosis transmembrane conductance regulator (CFTR) gene mutation associated with a congenital bilateral absence of vas deferens. Int J Urol 2008, 15(3):270-271.

26. Lu S, Yang X, Cui Y, Li X, Zhang H, Liu J, Chen ZJ: Different cystic fibrosis transmembrane conductance regulator mutations in Chinese men with congenital bilateral absence of vas deferens and other acquired obstructive azoospermia. Urology 2013, 82(4):824-828.

27. Shen Y, Liu J, Zhong L, Mogayzel PJ, Jr., Zeitlin PL, Sosnay PR, Zhao S: Clinical Phenotypes and Genotypic Spectrum of Cystic Fibrosis in Chinese Children. J Pediatr 2016, 171:269-276 e261.

28. Lee JH, Choi JH, Namkung W, Hanrahan JW, Chang J, Song SY, Park SW, Kim DS, Yoon JH, Suh Y et al: A haplotype-based molecular analysis of CFTR mutations associated with respiratory and pancreatic diseases. Hum Mol Genet 2003, 12(18):2321-2332.

29. Amaral MD: CFTR and chaperones: processing and degradation. Journal of molecular neuroscience : MN2004, 23(1-2):41-48.

30. Welsh MJ, Smith AE: Molecular mechanisms of CFTR chloride channel dysfunction in cystic fibrosis. Cell 1993, 73(7):1251-1254.

31. Hwang TC, Kirk KL: The CFTR ion channel: gating, regulation, and anion permeation. Cold Spring Harbor perspectives in medicine 2013, 3(1):a009498.

32. Lakdawala NK, Winterfield JR, Funke BH: Dilated cardiomyopathy. Circ Arrhythm Electrophysiol 2013, 6(1):228-237.

33. Reddy MM, Quinton PM: Functional interaction of CFTR and ENaC in sweat glands. Pflugers Arch 2003, 445(4):499-503.

34. Hume JR, Duan D, Collier ML, Yamazaki J, Horowitz B: Anion transport in heart. Physiol Rev 2000, 80(1):31-81.

35. Duan DY, Liu LL, Bozeat N, Huang ZM, Xiang SY, Wang GL, Ye L, Hume JR: Functional role of anion channels in cardiac diseases. Acta Pharmacol Sin 2005, 26(3):265-278. 
36. Sellers ZM, De Arcangelis V, Xiang Y, Best PM: Cardiomyocytes with disrupted CFTR function require CaMKII and $\mathrm{Ca}(2+)$-activated $\mathrm{Cl}(-)$ channel activity to maintain contraction rate. $J$ Physiol 2010 , 588(Pt 13):2417-2429.

37. Sellers ZM, Kovacs A, Weinheimer CJ, Best PM: Left ventricular and aortic dysfunction in cystic fibrosis mice. J Cyst Fibros 2013, 12(5):517-524.

38. Wallace CK, Bright LA, Marx JO, Andersen RP, Mullins MC, Carty AJ: Effectiveness of Rapid Cooling as a Method of Euthanasia for Young Zebrafish (Danio rerio). J Am Assoc Lab Anim Sci 2018, 57(1):58-63.

39. Matthews M, Varga ZM: Anesthesia and euthanasia in zebrafish. ILAR J 2012, 53(2):192-204.

40. Peng X, Li G, Wang Y, Zhuang J, Luo R, Chen J, Chen F, Shi Y, Li J, Zhou Z et al: CXXC5 is required for cardiac looping relating to TGFbeta signaling pathway in zebrafish. Int J Cardio/2016, 214:246-253.

41. Choudhry P, Trede NS: DiGeorge syndrome gene tbx 1 functions through wnt11 $r$ to regulate heart looping and differentiation. PLOS One 2013, 8(3):e58145.

42. Liu M, Liao H, Chen Y, Lin Z, Liu Y, Zhang X, Chan HC, Sun H: Treatment of human T-cell acute lymphoblastic leukemia cells with CFTR inhibitor CFTRinh-172. Leuk Res 2019, 86:106225.

\section{Figures}


Treatment of CFTRinh-172 $(10 \mu \mathrm{m})$ at 6-8 $\mathrm{hpf}$

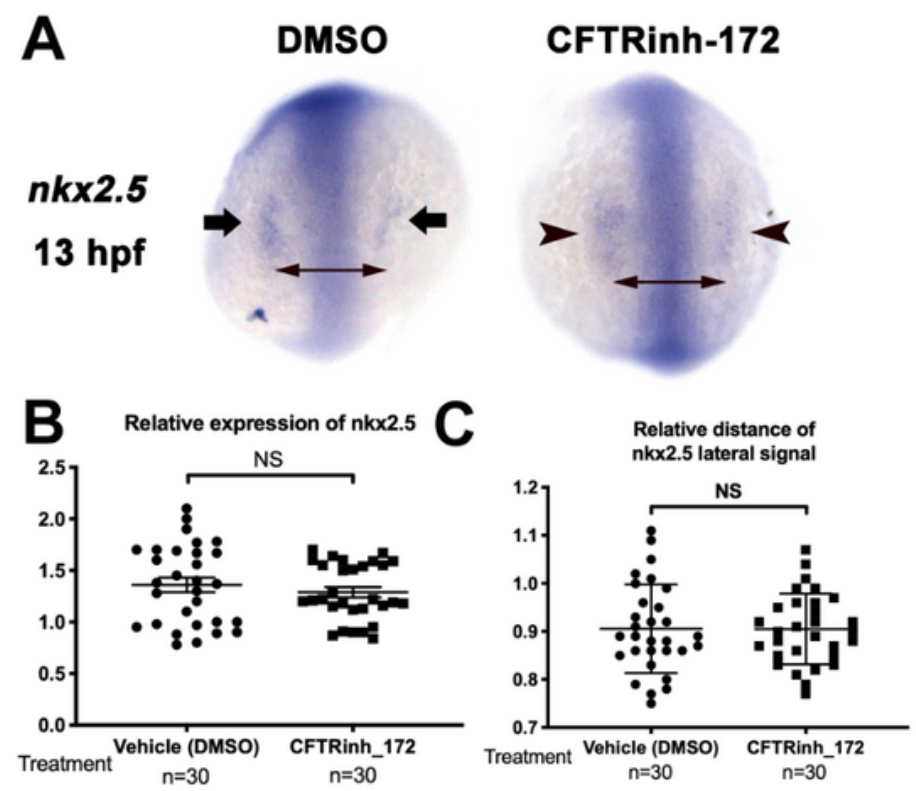

Treatment of CFTRinh-172 $(10 \mu \mathrm{m})$ at $8-10 \mathrm{hpf}$

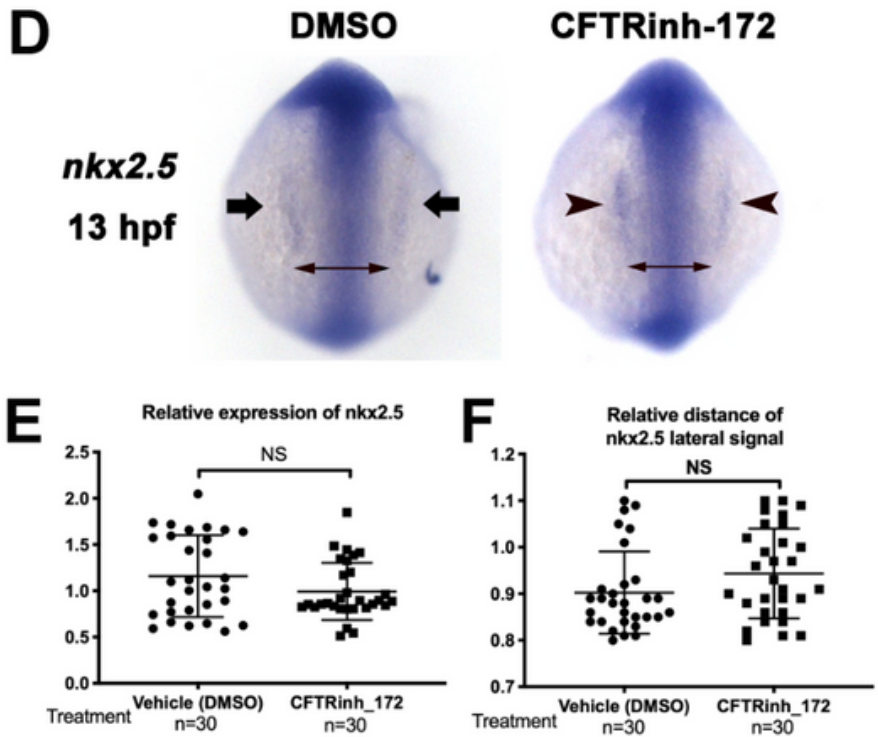

\section{Figure 1}

cftr mutation induces cardiac dysplasia in the early zebrafish embryo. (A) Lateral views of live zebrafish embryos at $60 \mathrm{hpf}$. Arrows indicate the pericardium. Heart rate (B) and quantification of ventricular fractional shortening (FS) (C) are represented in the scatter diagram. (D) The expression pattern of nkx2.5 at $13 \mathrm{hpf}$ is significantly affected in cftr mutant embryos. Embryo orientations: dorsal view with anterior oriented at the top. Arrows indicate normal nkx2.5 expression; arrowheads demonstrate the aberrant 
strength and position of nkx2.5 expression in the cftr mutant; double-ended arrows indicate the distance between the two populations expressing nkx2.5. Relative expression of nkx2.5 (E) and relative distance of nkx2.5 lateral signal $(F)$ are represented in the scatter diagram. (G) Heart morphology (marked by the pancardiomyocyte marker myl7) at $48 \mathrm{hpf}$ was impaired significantly in cftr mutants. Embryo orientation: ventral view with the anterior at the top. Arrows point to the atrium; red lines demonstrate the acute angle formed between the atrial and ventricular axes. Looping angle $(\mathrm{H})$ and area ratio of atrium/ventricle (I) are represented in the scatter diagram. The expression of bmp4 ( $\mathrm{J}$ and $\mathrm{K})$ and has2 $(\mathrm{L}$ and $\mathrm{M})$ is dramatically reduced in cftr mutant embryos at $48 \mathrm{hpf}$. Embryo orientation: ventral views with the anterior at the top. Arrows point to the signal generated by detected marker genes. 


\section{Treatment of CFTRinh-172 $(10 \mu \mathrm{m})$ at 3-5 hpf}
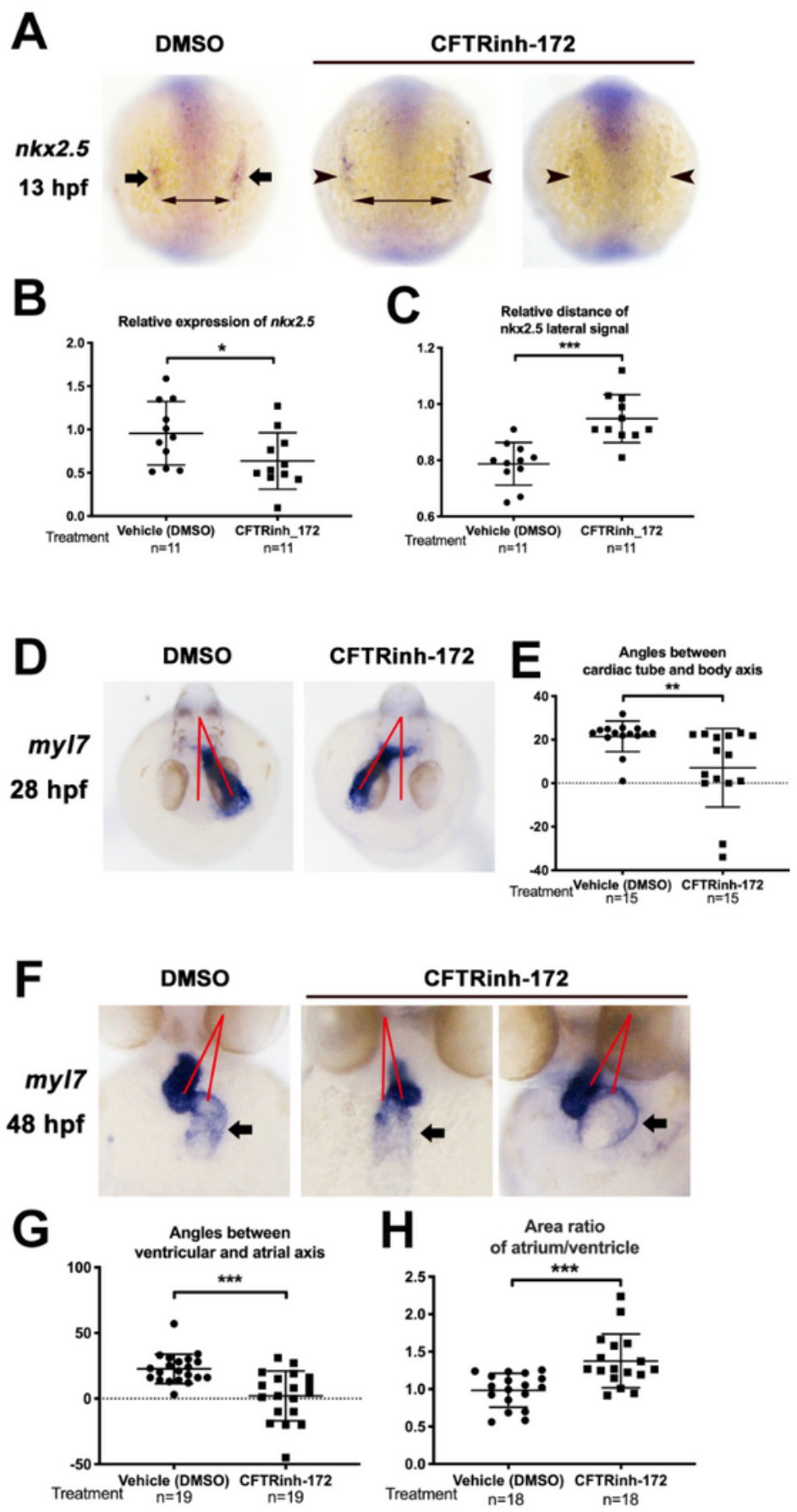

\section{Figure 2}

Treatment with the CFTR inhibitor CFTR_inh172 at the blastula stage leads to cardiac dysplasia in early zebrafish embryos. (A-C) The expression pattern of nkx2.5 at $13 \mathrm{hpf}$ is significantly affected. Embryo orientations: dorsal view with anterior oriented at the top. Arrows indicate normal nkx2.5 expression; arrowheads demonstrate the aberrant strength and position of nkx2.5 expression in the cftr mutant; double-ended arrows indicate the distance between the two populations expressing nkx2.5. (D and E) 
Heart morphology (marked by the pan-cardiomyocyte marker myl7) at $24 \mathrm{hpf}$ was altered significantly. Embryo orientation: ventral view with the anterior at the top. Red lines demonstrate the acute angle formed between the head midline and heart tube. (F-H) Heart morphology (marked by myl7) at $48 \mathrm{hpf}$ was impaired significantly. Embryo orientation: ventral view with the anterior at the top. Arrows point to the atrium; red lines demonstrate the acute angle formed between the atrial and ventricular axes.

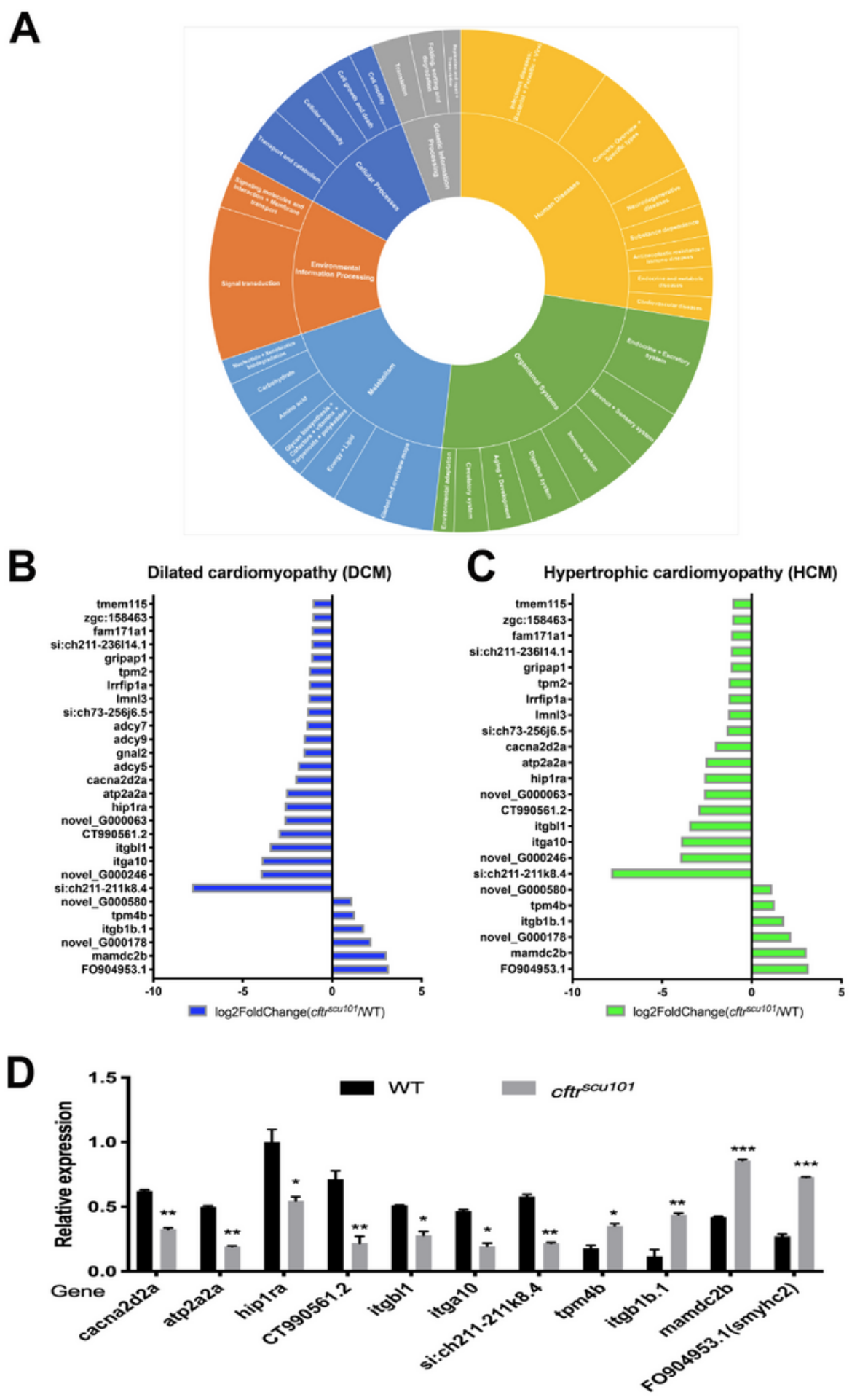

Figure 3 
Mutation of cftr induces significant alterations in transcription in blastula-stage embryos. (A) KEGG pathway classification analysis of transcriptomic data. KEGG analysis for dilated cardiomyopathy (DCM) (B) and hypertrophic cardiomyopathy (HCM) (C) gene sets using upregulated or downregulated genes comparing cftr mutant embryos with WT. (D) Key molecules of DCM and HCM affected by cftr mutant were verified by real-time PCR in embryos. The data represent the mean \pm SD from three experiments. ${ }^{\mathrm{P}}<$ $0.05,{ }^{\star} * \mathrm{P}<0.01$ vs WT.

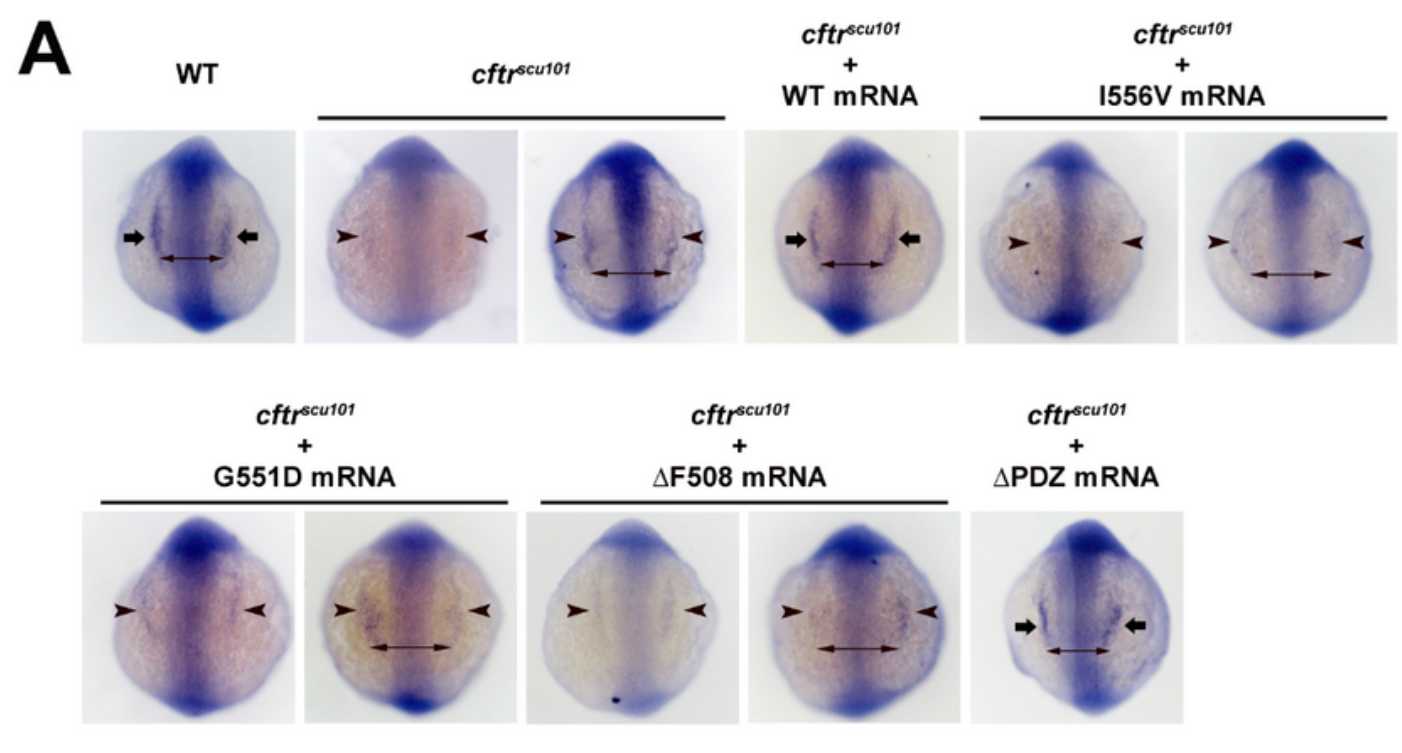

B

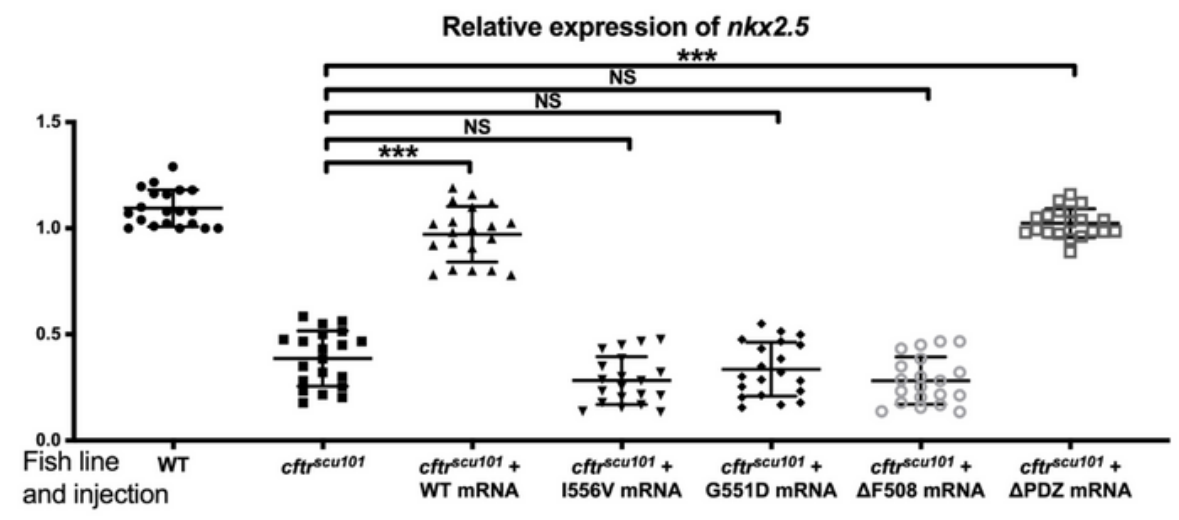

C

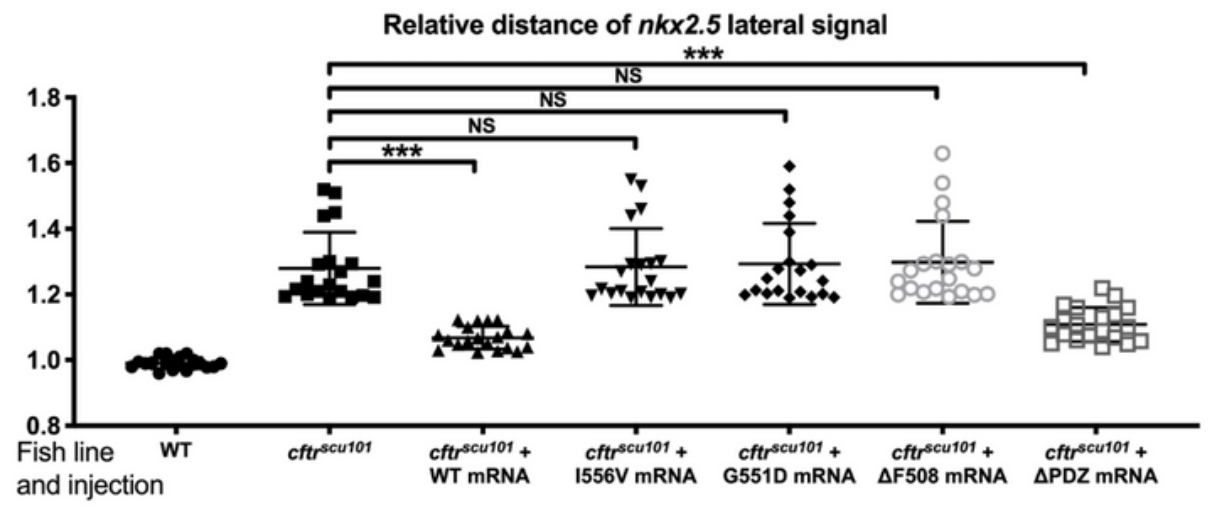

Figure 4 
CFTR I556V mutants fail to rescue nkx2.5-expressing cardiac progenitor cells at the 8-somite stage (13 hpf) of embryogenesis. (A) Embryo orientations: dorsal view with anterior at the top. Arrows point to the normal nkx2.5 signal; arrowheads demonstrate the aberrant strength and position of nkx2.5 expression induced by cftr mutants; double-ended arrows indicate the distance between the two nkx2.5+ progenitor populations. Relative expression of $n k x 2.5$ (B) and relative distance of nkx2.5 lateral signal (C) are represented in the scatter diagram.

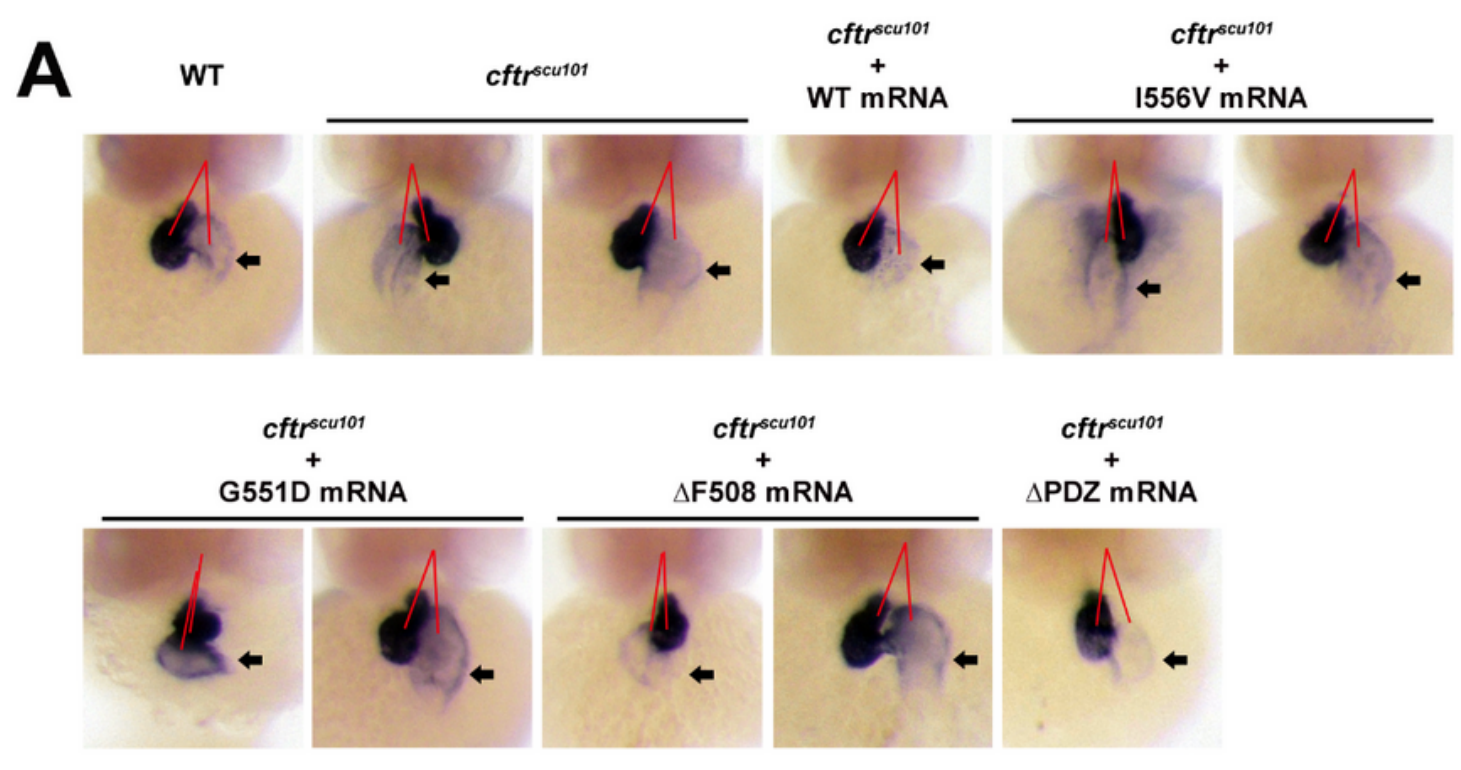

B
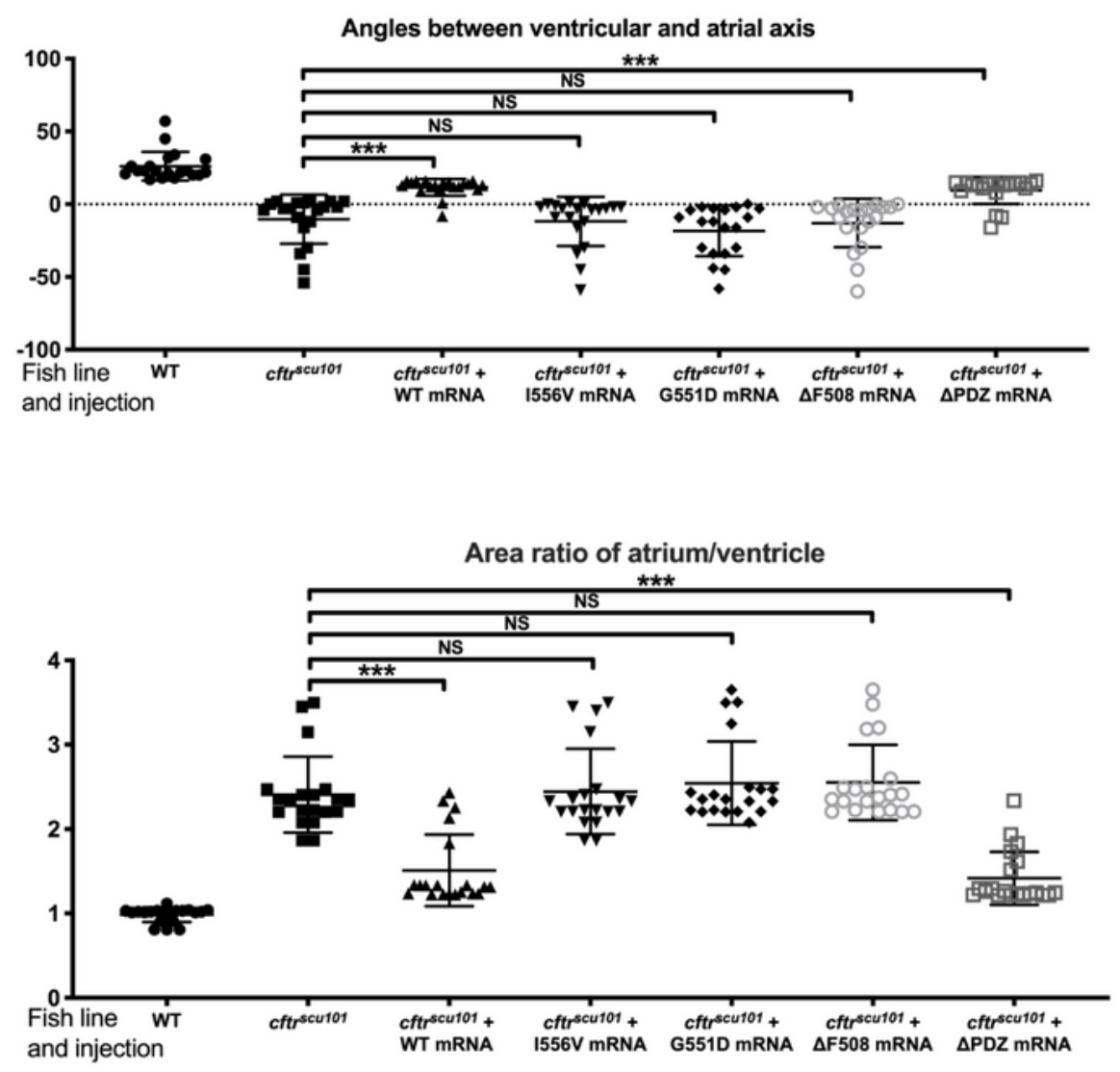

Figure 5 
CFTR 1556V fail to rescue heart morphology marked by myl7 expression 48 hpf during embryogenesis. (A) Embryo orientations: ventral views with the anterior at the top. Arrows point to the atrium; red lines indicate the acute angle formed between the atrial and ventricular axes. Looping angle (B) and area ratio of atrium/ventricle $(\mathrm{C})$ are represented in the scatter diagram.

A

Heart rate

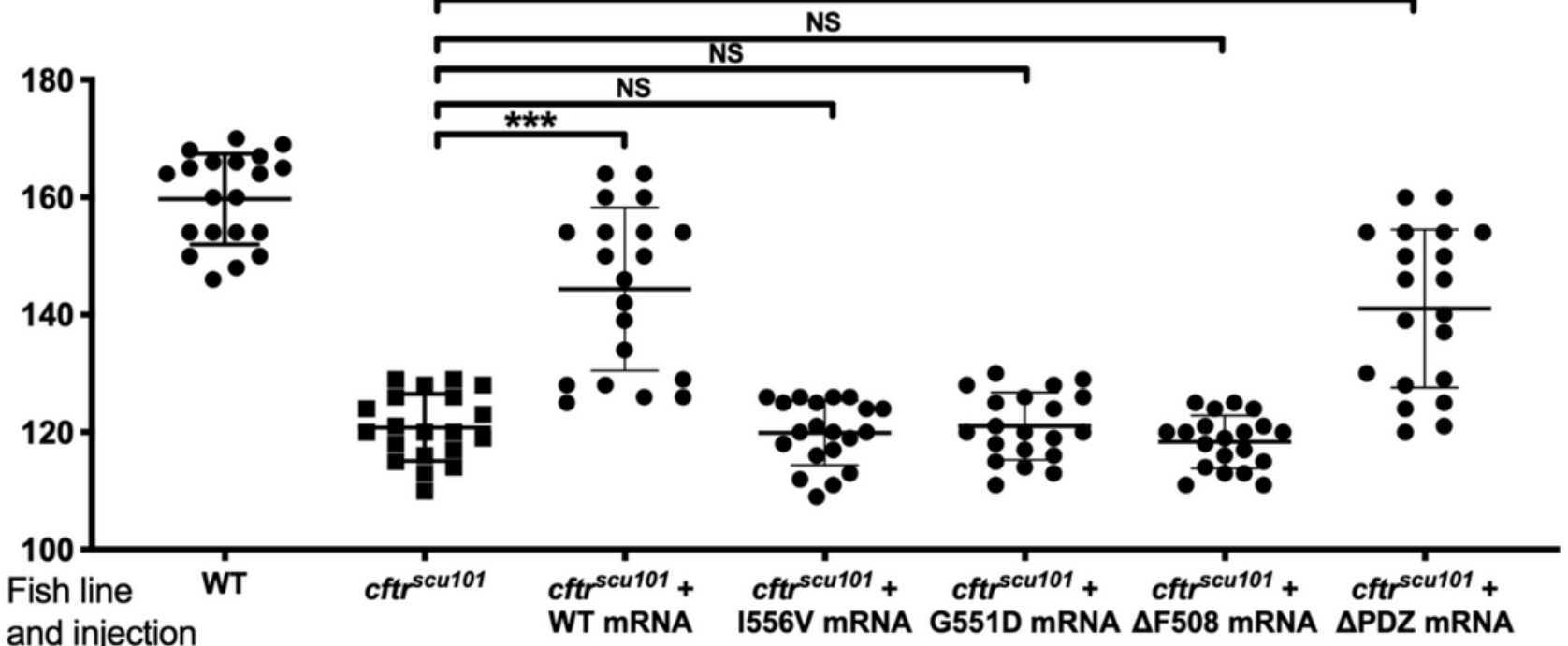

B

Fractional shortening (FS) (\%)

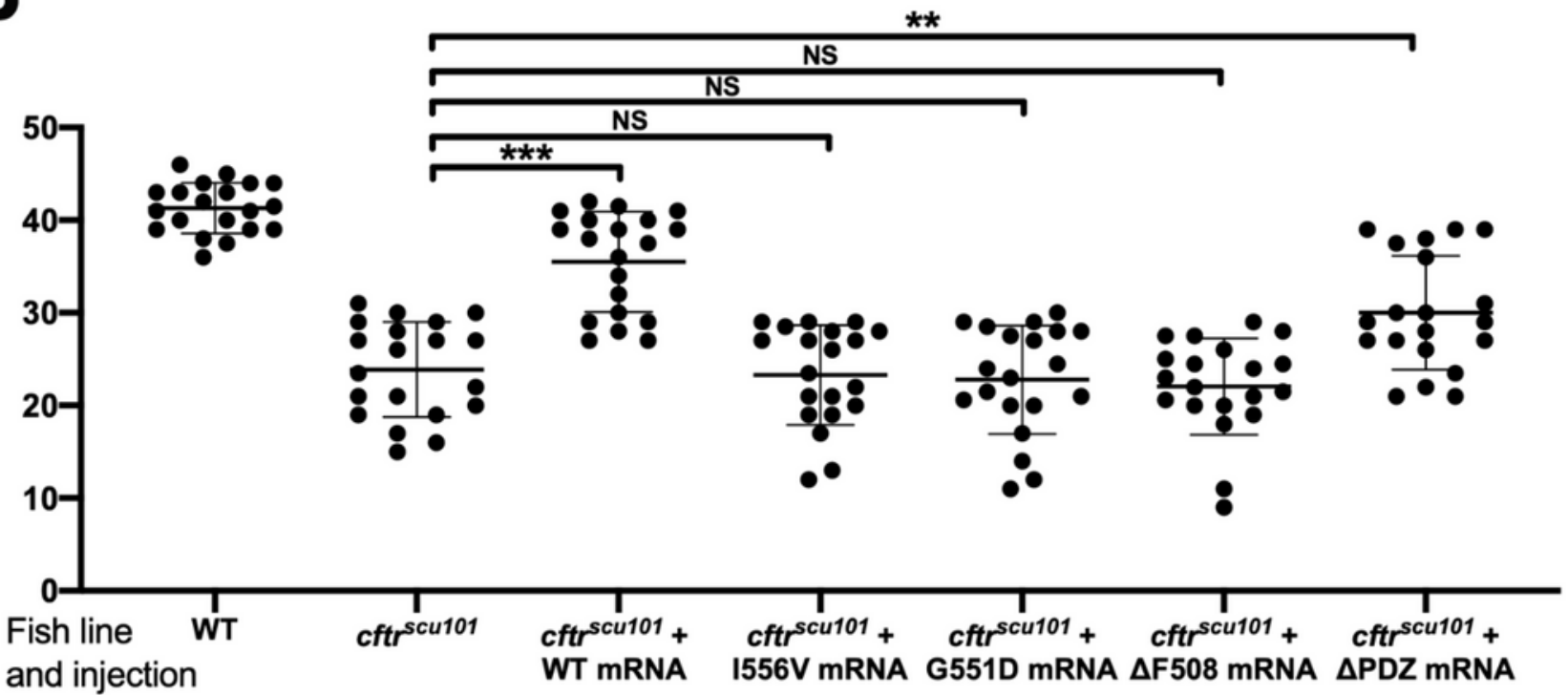

Figure 6 
CFTR I556V fail to rescue heart function at $60 \mathrm{hpf}$ during embryogenesis. Heart rate (A) and quantification of ventricular fractional shortening (FS) (B) are represented in the scatter diagram.

\section{Supplementary Files}

This is a list of supplementary files associated with this preprint. Click to download.

- Figs2.tif

- TableS101.xlsx

- ChecklistHuaqinSun.pdf

- TableS3DCMgenes01.xlsx

- TableS4HCMgenes01.xlsx

- TableS501.docx

- MSofCFTRheartSUP.doc

- TableS2.xIsx 